\title{
ESPECIES DE THYSANOPTERA EN UNA LOCALIDAD DEL CERRO DE LA MUERTE EN COSTA RICA, CENTRO AMÉRICA
}

\section{Gerardo A. SOTO-RODRÍGUEZ ${ }^{1,2}$, Axel P. RETANA-SALAZAR ${ }^{1,3}$ y Jesús A. RODRÍGUEZ-ARRIETA ${ }^{1,4}$}

\author{
${ }^{1}$ Centro de Investigación en Estructuras Microscópicas (CIEMIC), Ciudad de la Investigación, Universidad de \\ Costa Rica 2060, San Pedro de Montes de Oca, San José, Costa Rica \\ ${ }^{2}$ Hypericum Pharma, S.A. San José, Costa Rica. Apartado 1237-2050. cultivosyasesorias@yahoo.com \\ ${ }^{3}$ Escuela de Nutrición, Facultad de Medicina, Ciudad de la Investigación, Universidad de Costa Rica 2060, San \\ Pedro de Montes de Oca, San José, Costa Rica \\ ${ }^{4}$ Escuela de Biología, Universidad de Costa Rica 2060, San Pedro de Montes de Oca, San José, Costa Rica.
}

Recibido: 31/07/2014; aceptado: 20/11/2014

\begin{abstract}
Soto-Rodríguez, G. A., Retana-Salazar, A. P. \& Rodríguez-Arrieta, J. A. 2015. Especies de Thysanoptera en una localidad del Cerro de la Muerte en Costa Rica, Centro América. Acta Zoológica Mexicana (n. s.), 31(1): 84-88.
\end{abstract}

RESUMEN. El estudio se llevó a cabo de Julio 2010 a Octubre 2013 en 10 hectáreas localizadas en el Cerro de la Muerte, Costa Rica, Centroamérica. Se tomaron muestras mensuales por el método de golpeo de la vegetación en 22 especies de plantas, de las cuales sólo en 18 se obtuvieron thrips. Se recolectaron 14 especies de Thysanoptera, de las cuales 8 fueron de Terebrantia y 6 de Tubulifera. La especie que más se recolectó fue Liothrips sp. (24,4\%) y la asociada a un mayor número de plantas, Leptothrips astutus. El género de mayor incidencia fue Frankliniella, con cuatro especies. Se incluyen notas biológicas de las especies recolectadas.

Palabras clave: Empalme, Thysanoptera, especies, Terebrantia, Tubulifera.

\section{INTRODUCCIÓN}

Costa Rica cuenta con áreas de alta diversidad y endemismo en las tierras con elevaciones intermedias a altas a lo largo de eje montañoso principal (la región de Talamanca y la Cordillera Volcánica Central), así como en otros sitios con elevaciones medias a bajas y topografía irregular, entre ellos la Isla del Coco y las regiones del Golfo Dulce y Pacífico Central (Benavides 2008). El sitio de este estudio, el Cerro de la Muerte, es parte de la Ciudad de San José, ubicada en el Valle Central a $1200 \mathrm{msnm}$. Pasa de la Vertiente Pacífica a la Atlántica hacia la ciudad de Cartago, cruza el valle de El Guarco y asciende por las faldas de las estribaciones de la Cordillera de Talamanca, hasta llegar a la cima del Cerro a $3491 \mathrm{msnm}$ de altitud (Kappelle \& Omme 2000).

El Orden Thysanoptera es particularmente diverso en el Neotrópico donde las especies se alimentan en una variedad de sustratos (Mound 2002). Inicialmente (Mound \& Marullo 1996) hablaban de un total de 1600 especies descritas para Centro y Sur América. Sin embargo, diversos estudios realizados en más de 10 años han permitido
Soto-Rodríguez, G. A., Retana-Salazar, A. P. \& Rodríguez-Arrieta, J. A. 2015. Thysanoptera species in a locality of the Cerro de la Muerte in Costa Rica, Central America. Acta Zoológica Mexicana (n. s.), 31(1): 84-88.

ABSTRACT. The study was carried out from July 2010 to October 2013 on 10 hectares located in the Cerro de la Muerte, Costa Rica, Central America. We collected monthly samples by the method of beaten vegetation in 22 species of plants only from 18 we obtained thrips. 14 species of Thysanoptera were collected, of these 8 were Terebrantia and 6 Tubulifera. The species most collected was Liothrips sp $(24,4 \%)$ and associated to a greater number of plants, Leptothrips astutus. The genus of greater incidence was Frankliniella, with 4 species. Biological notes of the collected species are included.

Key words: Empalme, Thysanoptera, species, Terebrantia, Tubulifera.

incrementar el conocimiento de las especies (Soto-Rodríguez 2011) y generar nueva y valiosa información sobre Thysanoptera, en Centroamérica principalmente Costa Rica (Soto-Rodríguez \& Retana-Salazar 2013).

Mound \& Marullo (1996) han establecido que hay una alta diversidad de especies en las zonas altas de Costa Rica, siendo particularmente rica la región del Valle de los Santos, que se halla limitada parcialmente por el principio de la Cordillera de Talamanca, donde que presentan las mayores alturas del país. El estudio de estas regiones ha sido muy limitado y este nuevo aporte, centrado en el estudio de una localidad de altura, nos permite tener un mayor alcance en la ecología del grupo, como ha sido hallado en anteriores publicaciones (Sánchez-Monge et al. 2011)

Es importante señalar que la región estudiada, a pesar de ser una zona de altura, en ella se encuentran una serie de cultivos de importancia para el país como son el café, el aguacate, ornamentales y hortalizas entre otros. El estudio de la biodiversidad de thrips de la región permite analizar las posibilidades de especies que puedan ser utilizadas como controles, aquellas que pueden tener un 
rol como polinizadores y las que pueden presentar importancia por su potencial como plagas. El impacto de estos estudios acerca de la biología y la ecología de grupos con importancia económica van más allá de la mera inspección biológica, sino que presentan una importancia real e inmediata.

El objetivo de este trabajo fue identificar las especies de thrips asociadas a especies vegetales presentes en una localidad del Cerro de la Muerte en Costa Rica, Centro América y comentar sobre su biología.

\section{MATERIAL Y MÉTODOS}

Se realizaron muestreos mensuales desde Julio de 2010 hasta Octubre de 2013 en una superficie de 10 hectáreas ubicadas en San José, Cerro de La Muerte, específicamente en la localidad de El Jardín de Dota, a 1,5 km del Empalme, a $2195 \mathrm{msnm}$ de altitud y cuyas coordenadas geográficas son $09^{\circ} 43.170^{\prime} \mathrm{N}, 083^{\circ} 57.945 \mathrm{~W}$ (Fig. 1); la zona de vida de acuerdo a Bolaños et al. (2005) se reconoce como Bosque muy Húmedo Montano Bajo (bmh-MB). Esta zona presenta vegetación muy variada, en cuanto a árboles predominan el roble, el ciprés y el magnolio y hay numerosas plantas arbustivas y de porte bajo. Durante el período se muestrearon las diferentes plantas presentes en la zona de estudio mediante el método de golpeo de la vegetación (Cambero-Campos et al. 2010), que involucró a 22 especies vegetales.

\section{Preservación y montaje de especímenes}

Los thrips recolectados fueron almacenados en viales conteniendo alcohol etílico al 95\% y una etiqueta con el código del recolector y número consecutivo, con anotaciones de campo. Posteriormente el material se llevó al



Figura 1. Mapa de Costa Rica, Centro América, con la ubicación del sitio de estudio (estrella). laboratorio del Centro de Investigaciones en Estructuras Microscópicas de la Universidad de Costa Rica, donde fue procesado de acuerdo a las metodologías de montaje de microinsectos descritas por Walker \& Crosby (1988). Los especímenes fueron montados en láminas con Bálsamo de Canadá. El material de referencia se encuentra depositado en la Colección Institucional de Thysanoptera del CIEMIC de la Universidad de Costa Rica.

\section{Muestras botánicas}

Muestras de las especies vegetales con flor y/o fruto fueron recolectadas en campo y colocadas en bolsas plásticas de 30 x $40 \mathrm{~cm}$ y con la respectiva etiqueta que la asocia al código del vial del thrips presente en la planta. En el laboratorio, las muestras se prensaron en papel periódico y se colocaron en una prensa y en un secador. La identificación de las mismas se llevó a cabo utilizando la colección de referencia del Herbario de la Escuela de Biología de la Universidad de Costa Rica y del Herbario del Museo Nacional de Costa Rica. Además se contó con la ayuda del Ingeniero Olman Alvarado-Rodríguez para la determinación y preparación del material.

\section{RESULTADOS}

Las 22 especies vegetales (Cuadro 1) muestreadas pertenecen a 16 familias, de las cuales sólo en 18 especies se encontraron thrips. En total se contabilizaron 369 especímenes identificando 14 especies de Thysanoptera, 8 especies de Terebrantia y 6 de Tubulifera (Cuadros 3 y 4). La familia Asteraceae tuvo mayor incidencia de thrips con 6 especies (Cuadro 2) y dentro de esta familia, Ageratina altissima tuvo la mayor incidencia con 4 especies (Cuadro 3). En las familias Melastomataceae y Rosaceae se registraton 3 especies de thrips y las restantes familias de plantas tuvieron una incidencia menor a dos especies de thrips (Cuadro 2).

Leptothrips astutus fue el tisanóptero que se encontró asociado a un mayor número de plantas, concretamente a cinco especies (tres de Melastomataceae), lo cual coincide con los resultados de Johansen (1987) quien la encontró en plantas de varias familias. Asimismo, Sánchez-Monge et al. (2011) obtuvieron esta especie en plantas de Asteraceae y Verbenaceae.

A Liothrips sp., se le encontró asociada a cuatro especies vegetales (dos de Asteraceae) (Cuadro 3). Sánchez-Monge et al. (2011) afirman que Individuos de este género se han recolectado en otras plantas de la familia Asteraceae como Synedrella nodiflora comentando también que se conoce poco de los hospederos de este género en América Central. Esta especie fue la más abundante, 
Cuadro 1. Especies vegetales presentes en el sitio de estudio del Cerro de la Muerte en Costa Rica, Centro América.

\begin{tabular}{ll}
\hline Familia & Nombre científico \\
\hline Araliaceae & Schefflera rodriguesiana \\
Asteraceae & Ageratina altissima \\
& Cirsium subcoriaceum \\
& No identificada \\
& Senecio sp. \\
Bromeliaceae & Vriesea sp. \\
Dennstaedtiaceae & Pteridium sp. \\
Fagaceae & Quercus sp. \\
Gunneraceae & Gunnera insignis \\
Magnoliaceae & Magnolia sp. \\
Melastomataceae & Melastoma sp. \\
& Miconia sp. \\
Myricaceae & Monochaetum vestitum \\
Myrtaceae & Myrica cerífera \\
Onagraceae & Myrcianthes sp. \\
Phytolaccaceae & Fuchsia paniculata \\
Piperaceae & Phytolacca rugosa \\
Rosaceae & Piper sp. \\
Solanaceae & Eryobotria japonica \\
Winteraceae & Holodiscus argenteus \\
\hline
\end{tabular}

ya que se registraron 90 individuos (Cuadro 4) y varios inmaduros, resultando en $24,4 \%$ del total de thrips recolectados en el estudio, la mayoría obtenidos en Cirsium subcoriaceum en diferentes épocas del año, lo que hace suponer una asociación como hospedero entre planta y thrips, donde el insecto completa su ciclo de vida y no solo una simple relación como insecto visitante ocasional de esta especie vegetal.

De Leptothrips astutus se recolectaron 86 especímenes (23,6\%), registrando estados inmaduros solo en Monochaetum vestitum. Además de esta especie depredadora, se encontró un individuo de Franklinothrips lineatus, lo que coincide con Mound \& Reynaud (2005) en cuanto al estudio de un espécimen hembra de Costa Rica.

Cinco especies (3 de Tubulifera y 2 de Terebrantia) fueron las menos abundantes con porcentajes de entre 0,27-0,54\%. La presencia de estas especies raras por su baja abundancia (Gaston 1994, Guttman 1999), podría estar relacionada con sitios de mayor diversidad de hábitats, como ocurre en el Neotrópico, que son más ricos en número de especies, la mayoría son muy raras y endémicas (Retana-Salazar 2007).

De los géneros de thrips encontrados, el de mayor incidencia fue Frankliniella, del cual se encontraron cuatro especies, dos de las cuales son posiblemente nuevas y citadas como sp.1 y sp.2, F. annulipes se encontró en mayor porcentaje (Cuadro 4) y asociada a una sola especie vegetal (Cuadro 3). Del género Thrips, se encontraron dos especies (sp.1 y sp.2), siendo la especie asociada a Magnolia sp., la más abundante.

\section{DISCUSIÓN}

Información y datos biológicos de las diferentes especies de Thysanoptera encontradas en este estudio.

\section{TUBULIFERA}

\section{Adraneothrips alajuela Mound \& Marullo, 1996}

Esta especie fue descrita originalmente por Mound \& Marullo (1996) a partir de hembras recolectadas en zacates y ramas secas en las cercanías del Volcán Poás. En este trabajo se encontraron individuos solitarios en hojas colgantes secas en sitios sombreados.

Material recolectado: 12.vi.2013 (G.A. Soto) en hojas secas colgantes de Magnolia sp.; 03.vii.2013 (G.A. Soto) en hojas muertas colgantes de Ageratina altissima.

\section{Adraneothrips acutulus Mound \& Marullo, 1996}

Mound \& Marullo (1996) describieron esta especie de material recolectado en Zurquí de Moravia y además en el Cerro de la Muerte, al igual como se indica en este trabajo.

Material recolectado: 26.vi.2013 (G.A. Soto) en rama de Quercus sp y 03.vii.2013 (G.A. Soto) en hojas colgantes secas de un árbol de Melastomataceae no identificado. Las hojas vivas de este árbol presentaban numerosas aga-

Cuadro 2. Incidencia de especies de thrips por familia de planta en el sitio de estudio del Cerro de la Muerte en Costa Rica, Centro América.

\begin{tabular}{lc}
\hline Familia & Número de especies de thrips asociadas \\
\hline Araliaceae & 0 \\
Asteraceae & 6 \\
Bromeliaceae & 0 \\
Dennstaedtiaceae & 1 \\
Fagaceae & 1 \\
Gunneraceae & 1 \\
Magnoliaceae & 2 \\
Melastomataceae & 3 \\
Myricaceae & 0 \\
Myrtaceae & 1 \\
Onagraceae & 0 \\
Phytolaccaceae & 0 \\
Piperaceae & 1 \\
Rosaceae & 3 \\
Solanaceae & 1 \\
Winteraceae & 1 \\
\hline
\end{tabular}


Cuadro 3. Especie de thrips y la especie vegetal asociado en el sitio de estudio del Cerro de la Muerte en Costa Rica, Centro América.

\begin{tabular}{ll}
\hline Especie & Planta asociada \\
\hline Adraneothrips acutulus & Quercus sp. \\
& Drymis granadensis \\
& Melastomataceae n.i. \\
Adraneothrips alajuela & Magnolia sp \\
& Ageratina altissima \\
Liothrips sp. & Cirsium subcoriaceum \\
& Eryobotria japonica \\
& Myrcianthes sp. \\
& Senecio sp. \\
Leptothrips astutus & Melastoma sp. \\
& Melastomataceae n.i. \\
& Monochaetum vestitum \\
Pteridium sp. \\
Pseudophilothrips sp. & Piper sp. \\
Franklinothrips lineatus & Ageratina altissima \\
Bravothrips kraussi & Asteraceae n.i. \\
Frankliniella annulipes & Ageratina altissima \\
Frankliniella akaina & Solanum sp. \\
Frankliniella sp.1 & Holodiscus argenteus \\
Frankliniella sp.2 & Gunnera insignis \\
Thrips sp.1 & Ageratina altissima \\
\hline & Holodiscus argenteus \\
& Magnolia sp. \\
& Melastomataceae n.i. \\
\hline & \\
&
\end{tabular}

llas de Cecidomyiidae abandonadas, pero no se encontraron thrips inquilinos; 18.ix.2013 (G.A. Soto) en hojas colgantes muertas de Drymis granadensis.

\section{Liothrips sp. Uzel, 1895}

Durante el estudio se hicieron recolectas sucesivas en los hospederos abajo indicados, pero principalmente en Cirsium subcoriaceum, donde en la mayor parte de los muestreos se encontraron larvas, lo que hace suponer una posible asociación entre el thrips y esta especie vegetal que le sirve, en todo caso, para llevar a cabo su ciclo de vida.

Material recolectado: 5.vii.2010 (G.A. Soto) en hojas de Senecio sp.; 10.viii.2010 (G.A. Soto) en brotes de Cirsium subcoriaceum se recolectaron adultos y larvas; 17.viii.2010 (G.A. Soto) en las nervaduras de hojas maduras de Cirsium subcoriaceum; 12.x.2010 (G.A. Soto) en los tallos y hojas de Cirsium subcoriaceum; 19.viii.2011 (G.A. Soto) en árbol de Myrcianthes sp., solamente se encontraron en el lado sur del árbol y en la parte baja del follaje; 13.ix.2011 (G.A. Soto) en árbol de Myrcianthes sp.; 21.ix.2011 (G.A. Soto) en arbusto de Asteraceae; 17.viii.2010 (G.A. Soto) en las nervaduras de hojas maduras de Cirsium subcoriaceum; 20.iii.2012 (G.A. Soto) en Myrcianthes sp.; 28.viii.2013 (G.A. Soto) en hojas ma- duras de Cirsium subcoriaceum. Otro material: 26.ii.2004 (G.A. Soto) en brotes y hojas maduras de Cirsium subcoriaceum (Asteraceae).

\section{Leptothrips astutus Johansen, 1987}

Esta especie depredadora se encontró en forma abundante en las diferentes plantas muestreadas y señaladas abajo. Cabe destacar que se encontraron larvas únicamente cuando se hicieron muestreos en Monochaetum vestitum.

Material recolectado: 29.x.2010 (G.A. Soto) en hojas de planta no identificada; 03.viii.2011 (G.A. Soto) en hojas de planta no identificada. Las plantas se encuentran en un sitio con sombra parcial de árboles de ciprés y los thrips se recolectaron en la cara abaxial de las hojas, principalmente tiernas, entre las nervaduras cercanas al peciolo; 19.viii.2011 (G.A. Soto) en frondas de Pteridium y en hojas de Monochaetum vestitum; 21.ix.2011 (G.A. Soto) en arbusto de Asteraceae; 30.v.2012 (G.A. Soto) en el envés de las hojas de una Melastomataceae no identificada, creciendo a la orilla de una quebrada; 20.vi.2012 y 04.vii.2012 (G.A. Soto) en hojas de planta no identificada; 23.vii.2012 (G.A. Soto) en hojas de Monochaetum vestitum; 12.vi.2013 (G.A. Soto) en hojas de planta no identificada; 03.vii.2013 (G.A. Soto) en Monochaetum vestitum; 18.ix.2013 (G.A. Soto) en hojas de Piper sp. Otro material: 26.ii.2004 (G.A. Soto) en hojas de Melastoma sp., y de Monochaetum vestitum (Melastomataceae).

\section{Leptothrips columbianus Johansen, 1987}

Se recolectó un espécimen. Esta especie fue originalmente descrita por Johansen (1987) a partir de seis

Cuadro 4. Número y porcentajes de las especies de thrips encontradas en el sitio de estudio del Cerro de la Muerte en Costa Rica, Centro América.

$\begin{array}{lcc}\text { Especie } & \text { Número de individuos } & \mathbf{( \% )} \\ \text { Adraneothrips acutulus } & 12 & 3,25 \\ \text { Adraneothrips alajuela } & 2 & 0,54 \\ \text { Liothrips sp. } & 90 & 24,40 \\ \text { Leptothrips astutus } & 87 & 23,60 \\ \text { Leptothrips columbianus } & 1 & 0,27 \\ \text { Pseudophilothrips sp. } & 1 & 0,27 \\ \text { Franklinothrips lineatus } & 1 & 0,27 \\ \text { Bravothrips kraussi } & 21 & 5,69 \\ \text { Frankliniella sp.1 } & 8 & 2,17 \\ \text { Frankliniella sp.2 } & 25 & 6,77 \\ \text { Frankliniella akaina } & 1 & 0,27 \\ \text { Frankliniella annulipes } & 60 & 16,26 \\ \text { Thrips sp.1 } & 35 & 9,48 \\ \text { Thrips sp.2 } & 25 & 6,77 \\ \text { Total } & 369 & 100\end{array}$


especímenes procedentes de diferentes localidades de Colombia.

Material recolectado: 13.iii.2013 (G.A. Soto) en Ageratina altissima, en floración.

\section{Pseudophilothrips sp. Johansen, 1979}

Material recolectado: 19.viii.2011 (G.A. Soto). Varios individuos en arbusto de Asteraceae en el envés de las hojas, entre las nervaduras y algunos caminando sobre las hojas.

\section{TEREBRANTIA}

\section{Franklinothrips lineatus Hood, 1949}

Esta especie también fue recolectada por Mound \& Marullo (1996) en el Cerro de la Muerte, pero en trampa Malaise. Cambero-Campos et al. (2010) recolectaron más de 100 individuos de esta especie en Nayarit, México, asociada a arvenses y al cultivo de aguacate.

Material recolectado: 20.vi.2013 (G.A. Soto) en hojas de Ageratina altissima.

\section{Bravothrips kraussi Crawford, 1948}

Mound \& Marullo (1996) recolectaron esta especie en hojas de Solanum sp., y en flores de Datura sp.

Material recolectado: 22.ix.2010 y 12.x.2010 (G.A. Soto) en el envés de hojas maduras de plantas de Solanum sp. (Solanaceae).

\section{Frankliniella akaina Mound \& Marullo, 1996}

Material recolectado: 20.ii.2013 (G.A. Soto) en inflorescencia de Gunnera insignis

\section{Frankliniella sp.1}

Material recolectado: 13.iii.2013 (G.A. Soto) en Ageratina altissima, en floración.

\section{Frankliniella sp. 2 y F. annulipes}

Material recolectado: 25.iv.2013 (G.A. Soto). Se recolectaron numerosos adultos ( $\hat{\partial} \widehat{\partial} \mathrm{y}$ 우) en flores de $\mathrm{Ho}-$ lodiscus argenteus; ambas especies estaban en el mismo hospedero.

\section{Thrips sp.1}

Muy abundantes; se recolectaron adultos de ambos sexos, unos de color amarillo-anaranjado y otros de color café en flores semi abiertas y completamente abiertas. Se ubican en grupos en los pétalos y en la parte interna de la flor.

Material recolectado: 13.vi.2012 y 12.vi.2013 (G.A. Soto) en flores de árbol de Magnolia sp., (magnolio).

\section{Thrips sp.2}

Se recolectaron numerosos adultos ( $(+\uparrow$ y $\widehat{\jmath} \widehat{\jmath})$, unos de color negro y otros anaranjados, en la base de los estambres de la flor que comparten con numerosos curculiónidos de menos de $5 \mathrm{~mm}$ de longitud.

Material recolectado: 17.iv.2013 (G.A. Soto) en flores de árbol de Melastomataceae no identificado.
Agradecimientos. Al proyecto "Descripción y ultraestructura de los thrips de Mesoamérica (Thysanoptera: Insecta) y al proyecto "Estudio morfológico y genético de los estados inmaduros de thrips (Thysanoptera: Insecta) de relevancia económica en Hispanoamérica”, $\mathrm{N}^{\circ}$ 810-Bl-224. A la empresa Hypericum Pharma, S.A. por facilitar el muestreo en el área de estudio bajo su administración.

\section{LITERATURA CITADA}

Benavides, C. 2008. Efecto del ambiente en la distribución geográfica de plantas endémicas de Costa Rica y determinación de zonas aptas para la conservación. Tesis de Maestría, Universidad de Costa Rica, San José, Costa Rica.

Bolaños, R., Watson, V. \& Tosi, J. 2005. Mapa ecológico de Costa Rica (Zonas de Vida), según el sistema de clasificación de zonas de vida del mundo de L.R. Holdridge), Escala 1:750 000. Centro Científico Tropical, San José, Costa Rica.

Cambero-Campos, O. J., Johansen, R., Retana-Salazar, A. P., García, O., Cantú, M. \& Carvajal, C. 2010. Thrips (Thysanoptera) del aguacate (Persea americana) en Nayarit, México. Revista Colombiana de Entomología, 36: 47-51.

Gaston, K. J. 1994. Rarity. Chapman Hall. London. U.K. 205 pp.

Guttman, B. S. 1999. Biology. John Hopkins University Press. USA. 989 pp.

Johansen, R. M. 1987. El género Leptothrips Hood, 1909 (Thysanoptera: Phlaeothripidae) en el Continente Americano: Su sistemática, filogenia, biogeografía, biología, conducta y ecología. Monografías del Instituto de Biología de la Universidad Nacional Autónoma de México. 3:1-246.

Kappelle, M. \& Ommen van, L. 2000. Lista de la Flora Vascular de la Cuenca Superior del Río Savegre, San Gerardo de Dota, Costa Rica. Acta Botánica Mexicana (n.s), 51:1-38.

Mound, L. A. 2002. Thysanoptera biodiversity in the Neotropics. Revista de Biología Tropical, 50: 477-484.

Mound, L. A. \& Marullo, R. 1996. The thrips of Central and South America: An Introduction (Insecta: Thysanoptera). Memoirs on Entomology. 487 pp.

Mound, L. A. \& Reynaud, P. 2005. Franklinothrips; a pantropical Thysanoptera genus of ant-mimicking obligate predators (Aeolothripidae). Zootaxa, 864: 1-16.

Retana-Salazar, A. P. 2007. Estudio Taxonómico y Sistemático filogenético de los grupos genéricos Anactinothrips-Zeugmatothrips (Tubulifera: Idolothripinae). Editorial del Instituto Centroamericano para la Investigación en Biología y Conservación (CIBRC). Costa Rica.

Sánchez-Monge, A., Retana-Salazar, A. P., Brenes, S. \& Agüero, R. 2011. A contribution to thrips-plant associations records (Insecta: Thysanoptera) in Costa Rica and Central America. Florida Entomologist, 94: 330-339.

Soto-Rodríguez, G. A. 2011. Diversidad de Thrips (Thysanoptera) en Costa Rica. Métodos en Ecología y Sistemática, 6: 34-43.

Soto-Rodríguez, G. A. \& Retana-Salazar, A. P. 2013. Listado referencial de publicaciones sobre Thysanoptera para Costa Rica (1955-2012). Métodos en Ecología y Sistemática, 8: 5-24.

Walker, A. K. \& Crosby, T. K. 1988. The preparation and curation of insects. Information series 163, Entomology Division, Department of Scientific and Industrial Research, Auckland, New Zealand, Wellington. 92 pp. 